Leaver, T. (2015). Born Digital? Presence, Privacy, and Intimate Surveillance. In Hartley, John \& W. Qu (Eds.), Re-Orientation: Translingual Transcultural Transmedia. Studies in narrative, language, identity, and knowledge (pp. 149-160). Shanghai: Fudan University Press.

Chapter 10

\title{
Born Digital? Presence, Privacy, and Intimate Surveillance
}

Tama Leaver

Curtin University

\section{Introduction}

The moment of birth was once the instant when parents and others first saw their child in the world, but with the emergence of various imaging technologies, most notably the ultrasound, the first photos often precede birth (Lupton, 2013). In the past several decades, the question is no longer just when the first images are produced, but who should see them, via which, if any, communication platforms. Should sonograms (ultrasound photos) be used to announce the impending arrival of a new person in the world? Moreover, while that question is ostensibly quite benign, it does usher in an era when, for the first years of their life, parents and loved ones decide what, if any, social media presence young people have before they're in a position to start contributing to those decisions.

This chapter addresses this comparatively new online terrain, postulating the provocative term "intimate surveillance", which deliberately turns surveillance on its head, begging the question whether sharing affectionately, and with the best of intentions, can or should be understood as a form of surveillance. First, I examine the idea of co-creating online identities, touching on some of the standard ways of thinking about identity online, and then starting to look at how these approaches do and do not explicitly address the creation of identity for others, especially parents creating online identities for their kids. Then I review some ideas about surveillance and counter-surveillance with a view to situating these creative parental acts in terms of the kids and others being created. Finally, I explore several examples of parental monitoring, capturing and sharing of data and media about their children, using various mobile apps, contextualising these activities not with moral finger-wagging but by surfacing specific questions and literacies that parents may need to develop in 
order to use these tools mindfully, to ensure decisions made about their children's online presences are purposeful.

\section{Co-Creating Online Identities}

Within media and communications scholarship, there are some increasingly familiar ways of thinking about online identities. One popular frame is the concept of the networked self (Papacharissi, 2010), which explicitly acknowledges that online identities occur across multiple online platforms as amalgams, constituted via an array of social media tools. Writing on this topic, danah boyd (2010) offers the notion of networked publics, with four main characteristics:

- persistence: personal data does not necessarily decay online and may last forever;

- replicability: copies of networked digital information can be made easily, within or across different platforms;

- scalability: the difference between making one copy and a million is trivial and requires almost no additional effort;

- searchability: the way online identity-information is organised and accessed is, for most people, via a search tool or search engine such as Google.

Additionally, as almost all of these platforms are commercially operated, it is important to note that personal information is then corporately owned (Aufderheide, 2010).

Along similar lines, but from a software studies perspective, Anne Helmond (2010) articulates the notion of "Identity 2.0" with many similar characteristics to the networked self and networked publics. Two additional features that Helmond describes worth emphasising are: first, the idea that identity online is in perpetual beta, which means that the software platforms always demand more information, more updates, more photographs, and never suggest that identity online is complete; and second, that identity material online is often generated by other users, meaning that what individuals post about other people can and often does impact on the online sense of who they are, at times with even more impact than what users write about themselves. People are part of the always ongoing creative process of rendering online identities for themselves and others, in a co-creative loop.

The concept of web presence (Allen, 2009; Leaver, 2010) addresses the same identity terrain, but with a stronger focus on questions of agency and control. Web presence entails online footprints, which are largely in the control of the person they are about, and digital shadows, which, whether benignly or maliciously, are about, but not in the control of, a specific individual. Moreover, in this model, identity is described as a "social media river", which emphasizes that online identity is rapidly changing and can look quite different from different vantages, in part driven by the necessity of finding identities via different search engines. "Web presence" moves on from the 
Web 2.0 notion of user-generated content and, with Helmond, entails the argument that identity online is best understood as the realm of content-generated users.

The shared presumption by the networked self, Identity 2.0 , web presence and most other contemporary models of online identity, is that the agency of the user is central. There is a presumption that identity should be controlled, curated and managed by the self in question. If the individual is not in control, then that is seen as a problem to be fixed, whether it be through privacy settings, terms of use, rights or access. There is always a presumption that the agency of the individual being hailed into that process is paramount. Solutions involve more agency, more individualism, more control.

The question of identity control has become increasingly important in the past few years with the shift towards what is generally known as the "real-name web". Big online companies and platforms have been pushing hard to move away from a pseudonym-based system of online identity to one where real legal names are the default identities. Even where rare retreats are made from this generally hardline push, it is usually once identity databases are so dense and well reticulated that platforms like Facebook or Google can associate real names even when they are not directly used. This is all done in the service of providing advertisers with better access to targeted markets. As Liesbet van Zoonen describes the change:

Nowadays, however, the anonymity of the [early] Internet and the construction of online personas that do not reflect offline identities have been reconstructed as "risk factors" of internet use ... Governments, schools, parents and other concerned parties now routinely warn against online imposters, bullying and identity theft, and social network sites like Facebook or Google+ have policies requiring users to register with their real names and data, and prevent them from having more than one account. (van Zoonen, 2013: 45)

The debates around Google's shift to real names — dubbed the "nym wars" (Moll, 2014) — made it apparent that forcing everybody to use real names, even with the best of intentions, has real consequences that are at times harmful and problematic across the board. With a singular online identity, the question of agency or control becomes even more important when the legacy or history of that identity may be entirely inescapable.

Yet, for all the emphasis on agency, what happens before young people have the agency, literacy or skills to take the reins of their own selves online? Parents, guardians, loved ones and others inevitably set the initial identity parameters for young people online. Indeed, owing to medical imaging technologies, the first images of young people are often created, and often shared online, before they are born. Lupton describes ultrasound sharing as an increasingly normal part of contemporary pregnancy: 
The emergence of such social media platforms as Facebook, Flickr, Instagram, Twitter, Bundlr and YouTube facilitating the sharing of images has allowed the wide dissemination of imagery and information about the unborn in public forums. Indeed, sharing of the first ultrasound photograph on social media has become a rite of pregnancy for many women. (Lupton, 2013: 42)

Yet, as this rite of passage emerges, culturally there are no norms, no consistent ways of thinking through how and with whom these images should be shared. Moreover, for a generation who have become parents without direct experience of their own parents using social media, today's parents largely have to figure these rules out as they go. It may well be that such decisions only occur retrospectively, when children are old enough to turn around and demand as much control of their online presence as they can. How will they be positioned, how are parents today setting them up to be understood, and how will parents and children negotiate that process as they get older and want to start curating themselves? It is likely to be an argumentative process, a cocreative process; critically thinking that process through today may make some of the teen angst and generational anxieties of tomorrow slightly less fraught.

\section{Shifting Surveillance}

Beyond identity frameworks, when considering the way online information is captured, recorded, encoded and processed, it is also necessary to consider the contemporary landscape of surveillance. After revelations from WikiLeaks and Edward Snowden, it is all too obvious that contemporary society is deeply enmeshed in surveillance practices, whether that's physical and x-ray scans at airports, government tracking of both public and private data, the NSA attempting to track all social media and online activity via PRISM, loyalty card databases, or the more obviously visible examples of CCTV cameras peppered across metropolitan cities (Bodó, 2011; Lyon, 2014). On social media, surveillance occurs whether an individual uses a service or not: Facebook infamously revealed it keeps shadow profiles - ready-to-go profiles of potential users, gleaned from email addresses or other cues that show someone exists but is not yet using Facebook (Blue, 2013) - making the question of agency challenging at best. With layers of facial recognition being applied by Facebook and other platforms, simply being in the background of a photograph, even if no tags or text are added, can be enough to be interpellated into social media company databases (Althusser, 1971: 11; Oremus, 2014).

Yet surveillance is far from monolithic. More than a decade ago, notions of wearable technology were put forward as a means of inverting surveillance, the idea being that if devices are worn that survey the world from the wearer's point of view, this turns the process of watching on its head (Mann, Nolan, \& Wellman, 2002). Albrechtslund 
(2008) argues further that since surveillance practices are visible and well known, this allows individuals to treat being under surveillance as a form of knowing play, at times deliberately subverting what is recorded or captured. E. J. Westlake (2008) goes a step further, arguing that because a user's online performance and activity are being recorded, performances can be entirely false or misleading, subverting the effectiveness of even top-down surveillance. There is also the idea of sousveillance - organised watching of the watchers - seen in examples such as organised groups watching and reporting on Facebook's practices (Fernback, 2013), surveying the surveyors. There are even contemporary examples of physical resistance to the digital, where make-up can be applied in specific patterns that prevent facial recognition algorithms being able to actually "see" faces (Harvey, 2013).

Of course, subverting surveillance at any level usually requires active intervention. In contrast, Mark Andrejevic (2005) examines the terrain of peer surveillance, where individuals are encouraged actively to survey each other; to document, record and encode the actions of those around them. Peer surveillance is knowingly done, but by no means has to be driven by evil or malicious intent. Building on peer surveillance, then, where both the surveyor and the surveyed have agency to concede or resist on some level, I argue that where very young people are involved a more specific concept is needed, which I am calling "intimate surveillance".

Intimate surveillance involves the purposeful and routinely well-intentioned surveillance of young people by parents, guardians, friends, and so forth. The surveyed have little or no agency to resist. On one level, intimate surveillance points to the limits of most surveillance models, in that they are incomplete in trying to address subjects who have no agency or awareness of the means of resistance (for obvious reasons). An example of this is something as simple as sharing Facebook photographs of young children, tagging those photographs with their names, and thus having their identity exist as part of the Facebook database even if there is no named profile explicitly and publicly attached. While some parents do choose to fabricate a child's age and set up a Facebook profile for them from birth, even when this is not the case, tagging children's names on Facebook initiates the database building process, interpellating their existence at a generational level.

\section{Negotiating Intimate Surveillance}

The explosion in pregnancy and infant monitoring software, or apps for mobile devices, provides a solid foundation from which to view the increasing prevalence of intimate surveillance in contemporary networked culture. These apps are readily and easily downloadable to popular tablets and mobile phones. They usually have carefully designed minimalist interfaces, and make the process of documenting various cues to do with pregnancy and infancy very straightforward. In effect they normalise the 
process of everyday intimate surveillance. As an example, in the Apple app store there are hundreds of apps purporting to manage pregnancy or manage young life in some capacity.

The most popular pregnancy and infant apps at time of writing are from the Sprout brand. Sprout Pregnancy (http://medart-studios.com/sprout-pregnancy-iphone-app/), for example, encourages expectant mothers to track their weight, record when kicks are felt, and it even provides a timer for eventual contractions. Sprout Baby (http:// medart-studios.com/sprout-baby-iphone-ipad-app/) continues to encourage recording development, tracking various aspects of early life including the infant's weight, sleep and feeding. Over the space of days, weeks and months, their sleep patterns (if regularly entered into the app) can be displayed in terms of each day, each week, or each month, showing patterns for comparing to established norms. Feeding patterns can be recorded and mapped in a similar fashion, producing graphs and statistics that can be compared with established norms, or shared with others. It should be noted, of course, that recording such activities is far from new. Journalling weight, feeding and sleep has long been established in paper form, allowing medical oversight and intervention, or even just for the purposes of reassuring new parents that their child is developing normally. What is different now, though, is that every moment captured and recorded is stored in a corporate database. The terms of use of that corporate database are very carefully described, effectively stating that if other people access this information then you've already consented to that by putting your record into this database. Given the commercial imperatives to make the app profitable, the question of what happens to this information now, or in the future, is very unclear (DeNicola, 2012).

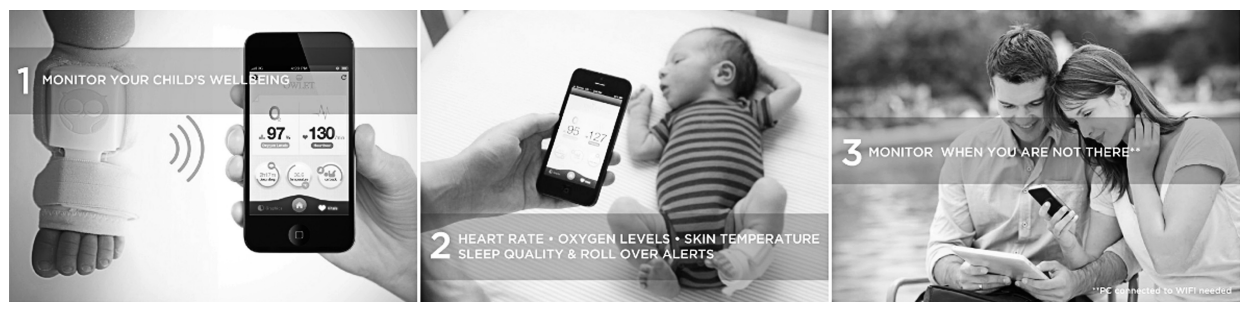

Figure 10.1: Owlet Care 2013 http://owletcare.com/

Combining an ankle bracelet with an app, the 2013 Owlet Care advertisement demonstrated a heart rate and blood oxygen monitor that infants could wear whenever they are asleep (see Figure 10.1). This wearable device removes the need for specific medical instruments to measure vital statistics, and can thus serve as an early warning system if something were to go wrong. However, the initial advertisement 
looked rather unappealingly like a ball and chain, immediately evoking questions of surveillance and control, not just parental concern. However, the thing about this advertisement that most stands out is the fact that the parents are watching the data generated from the app in a park, where clearly there is no baby in the photograph; this reduces the child's wellbeing to the data on a mobile device's screen, while the child is left at home, presumably with a caregiver of some sort, but there's a highly mediated and surveillance-driven idea of "enjoying" parenthood in this triptych. To be fair to the Owlet company, in their more recent model this advertisement has been discontinued, and the Owlet has been remodelled as a less invasive "smart sock", a surveillance bootee that looks more cuddly and sweet. It is indicative of an era where mobile devices, apps, and wearable technologies vastly increase the ability to monitor and record data about pregnancy and young children. Questions of when recording and sharing are appropriate thus become vitally important to ask today.

Drawing on a somewhat different use of mobile apps, and a different dataset, it is possible to track the use of specific types of images on the photo-sharing app Instagram that are related to pregnancy and infancy via specific hashtags (Highfield $\&$ Leaver, 2014). In our own research, the hashtag \#birth was tracked from the beginning of March 2014 to the end of May 2014, in order to ascertain how frequently Instagram users specifically and explicitly marked their photographs or videos with the term birth. The birth tag was filtered so that only "birth" was returned, not other words beginning with birth, such as birthday, which would have resulted in a much larger and less specific dataset. Over the three-month period, a total of 38,409 media items were found, comprising 36,812 images and 1,597 videos. Over the same period, the \#ultrasound hashtag was tracked, which showed that over the same three months 10,890 images and 430 videos were tagged, with a total of 11,320 media items. To drill down into the data qualitatively, all of the images for the first Monday of each month were separated out (each Monday effectively covered 48 hours, since it included anywhere in the world it might be Monday). Here, I'll focus on the data from the first Monday in March, which included 289 specifically tagged images and 7 \#ultrasound videos.

Some of the results were quite unexpected. For example, there were 8 examples of ultrasound humour, where image manipulation has occurred, which at times included a Starbucks coffee or a wad of cash in a foetal ultrasound image. There were 32 photos that can be categorised as the social experience of the ultrasound, such as parents in the car looking pensive and excited on their way to the first ultrasound scan. There were a further 32 images which included collages or professional photographs, a form of emergent digital storytelling, including collages with seven frames that narrate the story from the first home pregnancy indicator, to the first scan, to the second scan, to choosing a name, and then pensive faces before the infant is born.

However, the most notable finding in relation to the topic of this chapter is the 
fact that 71 photos or $26 \%$ of the set included personally identifiable information. Typically, foetal ultrasounds - or sonograms — generally include metadata in the picture; typical details include the mother's date of birth, the medical facility where the scan is taking place, the estimated due date, the date of the scan, and sometimes the doctor who referred the person being scanned. Linking back to the shift towards real name use online, and in the context of contemporary scanning technology where it takes trivial effort to read the text in a photograph (even if it's not in a text field in the metadata), these publicly-shared ultrasound photographs could easily be used to extrapolate the imminent arrival of a new family member, and thus allow companies that rely on profiles to begin a shadow profile even before that person is actually born. These images contain all of the information needed to infer a person, or a person who is coming into being.

\begin{tabular}{ll}
\hline 16 October 2010 & Instagram app launched via Apple's App Store \\
12 December 2010 & 1 million registered users
\end{tabular}

3 August 2011

September 2011

3 April 2012

9 April 2012

26 July 2012

16 August 2012

5 November 2012

5 December 2012

17 December 2012

18 December 2012

26 February 2013

20 June 2013

10 July 2013

6 September 2013

12 December 2013

24 March 2014

26 August 2014
150 million photos uploaded

10 million registered users

Instagram releases Android version

Facebook purchases Instagram for \$US1 billion

80 million registered users

Instagram Photo Maps launched

Instagram Profiles for the Web launched

Instagram removes ability for photos to appear as "cards" on Twitter

Instagram Alters Terms of Use

Instagram reverts to previous Terms of Use after public backlash

100 million active monthly users

Instagram adds video (15-seconds maximum)

Instagram adds native web embedding for photos and videos

150 million users

Instagram Direct messaging service added

200 million users

Instagram/Facebook release Hyperlapse app via Apple App Store

Figure 10.2: Instagram Development Timeline

The mobile creation and sharing of photographs and videos is especially interesting when thinking about the privacy minefield that is Instagram. Typically, Instagram 
is thought of in either/or terms; it's either private or public. However, as Fig. 10.2 demonstrates, Instagram's development is more complicated than that: it began as an app that a relatively small number of people could use or see as it was only available for the iPhone. Since then it has released versions for Android and Windows mobile operating systems, added maps that allow users easily to navigate embedded geographic data in many photos. It has released a web-based version where nonInstagram users can view profiles of users online, and it has added the ability for nonusers to embed Instagram photographs and videos elsewhere on the web. While most of these changes are not technical — in that material hasn't been made public that was once technically private - nevertheless the experience of privacy, or the expectation of a specific audience, has radically changed over time.

The shifting terrain of accessibility complicates the process so that even someone who is actively trying to think about the appropriateness of specific tools is not only subject to changes in the way that app or platform is accessed, but also to changes in terms of ownership. When Instagram was purchased by Facebook, the corporation immediately had access to all of Instagram's user data, and could easily link Facebook and Instagram accounts for many users. Facebook's fairly poor history of dealing with customer privacy (boyd, 2008; boyd \& Hargittai, 2010) understandably leads to a certain hesitation in trusting their management of Instagram. This tension is what Leaver and Lloyd (Leaver \& Lloyd, 2015, p.162) describe as a "social media contradiction":

A social media contradiction may arise where users focus on the social elements - often acts of communication and sharing which are thought of as ephemeral and in the moment, comparable to a telephone conversation - while the companies and corporations creating these apps are more focused on the media elements, which are measurable, aggregatable, can be algorithmically analysed in a variety of potentially valuable ways, and can last indefinitely.

Thus on the social side, the sharing of ultrasound photographs is a very conversational act, a form of communication that is often about sharing the joy and excitement about pregnancy and new life. Whereas the media side is the ongoing storage, analysis and mining of images and data that allow corporations to track and profile users, usually for the banal purposes of selling advertising, but always focused on that bottom line rather than user privacy or any other overriding concerns. While the use of the Sprout apps, or Owlet wearable device, are more obviously tracking and recording data, the sharing of ultrasound photographs on Instagram is instructive in that a similar level of recording and analytics is possible, but in manner that is even further removed from the attention of the average user.

While the Sprout, Owlet and Instagram examples illustrate intimate surveillance, 
it is important to reiterate that the motivations in recording and sharing media in these examples are far from sinister; almost always they are about taking care of others or sharing the excitement about new lives. Nor is it the case that all aggregations of data — "big data" - are inherently problematic. For example, in a controlled big data tracking study focused on prematurely born babies, using "software that captures and processes patient data in real time, tracking 16 different data streams, such as heart rate, respiration rate, temperature, blood pressure, and blood oxygen level, which together amount to around 1,260 points of data", revealed significant diagnostic correlations, leading to very real health improvements for those children (Mayer-Schoönberger \& Cukier, 2013: 60-61). That outcome could only have come through big observational data. In this form of study, though, it is important that it is transparent. It is controlled and parents have given informed consent, and that is vital. Parents, or whoever informed or gave permission, understood what they were doing and that was transparent and communicated to them. Intimate surveillance, on the other hand, is usually on the wrong side of the social media contradiction, wherein the actual uses of the data generated and recorded are often occluded and not transparently explained at all.

\section{Conclusion}

Intimate surveillance normalises a surveillance culture facilitated by our nearest and dearest, normalising the idea that parents will survey their kids in particular ways. This context requires the development of more robust digital literacies about the uses and potential abuses of data and metadata shared with apps, platforms, corporations and so forth. Terms of Use ideally need to explain not just the way personal information is currently used, but how else those data might be used. This is particularly important to make clear, given that Terms of Use are almost always written in legal jargon that few users can actually readily understand, even if they do have time to read them. Moreover, this is not a technology or data literacy problem. It is also a generational change, which begs the development of new social norms. Parents negotiating with other parents about what photographs of their children they do or do not want shared is but one example of the need for new discussions, new understandings, when every mobile phone is potentially not just a media creation device, but an instantaneous publication tool as well. For young people whose first online images precede their birth, they may indeed be born digital, but the cultural systems in which we understand and frame those images are evolving ones, which mix the lessons of analogue and digital eras.

\section{References}

Albrechtslund, A. (2008) Online social networking as participatory surveillance. First Monday, 
13(3). Retrieved from http:/firstmonday.org/htbin/cgiwrap/bin/ojs/index.php/fm/article/ view/2142/1949

Allen, M. (2009) Web Presence: Understanding persistent and interlinked content as the basis of identity formation and promotion through the contemporary Internet. Presented at the Communication, Creativity and Global Citizenship: Australia and New Zealand Communications Association Annual Conference, Brisbane.

Althusser, L. (1970) Lenin and Philosophy and Other Essays. London: Verso.

Andrejevic, M. (2005). The work of watching one another: Lateral surveillance, risk, and governance. Surveillance \& Society, 2(4), 479-497.

Aufderheide, P. (2010) Copyright, fair use, and social networks. In Z. Papacharissi (Ed.), $A$ networked self: identity, community, and culture on social network sites (pp.274-303). Routledge.

Blue, V. (2013, June 23) Anger mounts after Facebook's “shadow profiles” leak in bug. ZDNet. Retrieved from http://www.zdnet.com/anger-mounts-after-facebooks-shadow-profiles-leakin-bug-7000017167/.

Bodó, B. (2011) You have no sovereignty where we gather - Wikileaks and freedom, autonomy and sovereignty in the Cloud. SSRN eLibrary. Retrieved from http://papers.ssrn.com/sol3/ papers.cfm?abstract_id=1780519\#.

boyd, danah. (2008) Facebook's privacy trainwreck: Exposure, invasion, and social convergence. Convergence, 14(1), 13-20.

boyd, danah. (2010) Social network sites and networked publics: Affordances, dymanics and implications. In Z. Papacharissi (Ed.), A Networked Self: Identity, Community, and Culture on Social Network Sites (pp.39-58). Routledge.

boyd, danah, \& Hargittai, E. (2010). Facebook privacy settings: Who cares? First Monday, 15(8). Retrieved from http://www.uic.edu/htbin/cgiwrap/bin/ojs/index.php/fm/article/ view/3086/2589

DeNicola, L. (2012) EULA, Codec, API: On the Opacity of Digital Culture. In P. Snickars \& P. Vonderau (Eds.), Moving Data: The iPhone and the Future of Media (pp.265-277). New York: Columbia University Press.

Fernback, J. (2013) Sousveillance: Communities of resistance to the surveillance environment. Telematics and Informatics, 30(1), 11-21. doi: 10.1016/j.tele.2012.03.003

Harvey, A. (2013) CV Dazzle: Camouflage from face detection. Retrieved December 3, 2014, from http://cvdazzle.com/.

Helmond, A. (2010) Identity 2.0: Constructing identity with cultural software. Www. annehelmond.nl, PDF: http://www.annehelmond.nl/wordpress/wp-content/uploads//2010/01/ helmond_identity20_dmiconference.pdf.

Highfield, T., \& Leaver, T. (2014) A Methodology for mapping instagram hashtags. Presented at the Digital Humanities Australasia 2014, Perth, Australia. Retrieved from http://www. slideshare.net/Tama/a-methodology-for-mapping-instagram-hashtags

Leaver, T. (2010) I tweet therefore I am? Challenges in learning identity by teaching web presence. Presented at the Teaching and Learning Forum, Edith Cowan University, 
Joondalup.

Leaver, T., \& Lloyd, C. (2015) Seeking Transparency in Locative Media. In R. Wilken \& G.

Goggin (Eds.), Locative Media (pp.162-174). London \& New York: Routledge.

Lupton, D. (2013) The Social Worlds of the Unborn. Basingstoke: Palgrave MacMillan.

Lyon, D. (2014) Surveillance, Snowden, and Big Data: Capacities, consequences, critique. Big Data \& Society, 1(2), 2053951714541861. doi: 10.1177/2053951714541861

Mann, S., Nolan, J., \& Wellman, B. (2002) Sousveillance: Inventing and using wearable computing devices for data collection in surveillance environments. Surveillance \& Society, 1(3), 331-355.

Mayer-Schoönberger, V., \& Cukier, K. (2013) Big data: A revolution that will transform how we live, work, and think. Great B: John Murray.

Moll, E. (2014). What's in a Nym? Gender, race, pseudonymity, and the imagining of the online persona. M/C Journal, 17(3). Retrieved from http://journal.media-culture.org.au/index.php/ mcjournal/article/view/816

Oremus, W. (2014, March 20) Facebook facial recognition takes a giant, scary leap forward. WA Today. Retrieved from http://www.watoday.com.au/digital-life/digital-life-news/facebookfacial-recognition-takes-a-giant-scary-leap-forward-20140320-3547d.html.

Papacharissi, Z. (Ed.). (2010) A Networked Self: Identity, Community, and Culture on Social Network Sites. Routledge.

Westlake, E. J. (2008). Friend me if you Facebook: Generation Y and performative surveillance. TDR: The Drama Review, 52(4), 21-40.

Zoonen, L. van. (2013) From identity to identification: Fixating the fragmented self. Media, Culture \& Society, 35(1), 44-51. doi: 10.1177/0163443712464557 